Please do not remove this page

RMIT

UNIVERSITY

\title{
Level-crossing rate and average duration of fades for mobile radio channel with hyperbolically distributed scatterers
}

Shang, Lei; Mahmoud, Seedahmed; Hussain, Zahir

https://researchrepository.rmit.edu.au/esploro/outputs/9921859119001341/filesAndLinks?institution=61RMIT_INST\&index=null

Shang, L., Mahmoud, S., \& Hussain, Z. (2005). Level-crossing rate and average duration of fades for mobile radio channel with hyperbolically distributed scatterers. Proceedings of the 2005 IEEE Asia-Pacific Conference on Communications, 208-211. https://doi.org/10.1109/APCC.2005.1554049

Document Version: Published Version

Published Version: https://doi.org/10.1109/APCC.2005.1554049

Repository homepage: https://researchrepository.rmit.edu.au

(c) 2005 IEEE. Personal use of this material is permitted. However, permission to reprint/republish this material for advertising or promotional purposes or for creating new collective works for resale or redistribution to servers or lists, or to reuse any copyrighted component of this work in other works must be obtained from the IEEE.

Downloaded On 2023/04/26 20:14:01 +1000 


\title{
LeveL-Crossing Rate and Average Duration of Fades for Mobile Radio channel with Hyperbolically Distributed Scatterers
}

\author{
Lei Shang Student Member, IEEE, Seedahmed S. Mahmoud, Member, IEEE \\ and Zahir M. Hussain, Senior Member, IEEE \\ School of Electrical and Computer Engineering \\ RMIT University, Melbourne, Victoria 3000, Australia \\ Lei.Shang@ieee.org, seedahmed@ieee.org, zmhussain@ieee.org
}

\begin{abstract}
In this paper we study the geometrical and timevariant wireless vector channel model with hyperbolically distributed scatterers for a macrocell mobile environment. In this study we investigate the level-crossing rate (LCR), the average duration of fades (ADF), the probability density function (PDF), the cumulative distribution function (CDF) and the autocorrelation functions (ACF) of this recently-proposed model. The simulated results are verified against the analytical Clarke's channel model.In this paper we study the geometrical and time-variant wireless vector channel model with hyperbolically distributed scatterers for a macrocell mobile environment. In this study we investigate the level-crossing rate (LCR), the average duration of fades (ADF), the probability density function (PDF), the cumulative distribution function (CDF) and the autocorrelation functions (ACF) of this recently-proposed model. The simulated results are verified against the analytical Clarke's channel model.
\end{abstract}

\section{INTRODUCTION}

All relevant components of a mobile radio system, from digital modulation techniques over channel coding through to network aspects, are determined by the propagation characteristics of the channel. Therefore, a precise knowledge of mobile radio channels is crucial for the development, evaluation and test of current and future mobile radio communication systems.[1]

From the designers's viewpoint, simulation models for fading channels are extremely important for the development, performance analysis, and test of modern wireless communication systems. The designed fading channel simulator should fit the desired statistical behavior with high precision before analyzing the performance of a new mobile communication system. Accuracy, efficiency, flexibility and ease of implementation are the challenging requirements in designing the simulation models [2].

The characteristic quantities describing the statistics of mobile fading channels are the probability density function (PDF), cumulative distribution function (CDF), the autocorrelation function (ACF), the level-crossing rate (LCR) and the average duration of fades (ADF). The level-crossing rate (LCR) and average duration of fades (ADF) are useful for designing error control codes and diversity schemes to be used in mobile communication systems, since it becomes possible to relate the time rate of change of the received signal to the signal level and velocity of the mobile [3].

In [4], a space-time geometrical based hyperbolically distributed scatterers (GBHDS) model for a macrocell mobile environment was proposed. The combination of stochastic and geometrical assumptions results in a mathematically tractable and computationally efficient channel model. This model provides the power of each path, the time-of-arrival (TOA), and the direction-of-arrival (DOA) of the multipath component as well as the fading effect. The model enables the simulation of downlink beamforming as well as space diversity concepts and handles both spatially narrowband and wideband signals.

Verification of this newly proposed GBHDS model against the analytic fading channel model is thus necessary and a detailed analysis of the statistical characteristics of this new model is given.

The aim of this work is to study the GBHDS model and to analyze the LCR and ADF of this simulation model for Rayleigh fading channels. We first describe in Section II the stochastic reference model for the Rayleigh fading channel. In Section III, we briefly present the GBHDS model. Section IV presents the simulation results of the GBHDS simulator and the analysis of its statistical characteristics. The simulation results are compared with analytic results.

\section{Description of the Analytical Model}

The detailed derivation of Jakes Power spectral density or Clarke power spectral density can be found in [5]. We here review this derivation briefly and give some initial simulation results.

To derive the Jakes power spectral density, we follow these assumptions [1]:

1) The propagation of the electromagnetic waves takes place in the two-dimensional(horizontal) plane, and the receiver is located in the center of an isotropic scattering area.

2) The angles of arrival $\alpha$ of the waves arriving the receiving antenna are uniformly distributed in the interval $[-\pi, \pi)$. 
3) The antenna radiation pattern of the receiving antenna is circular-symmetrical (omnidirectional antenna).

The probability density function of the angles of arrival $\alpha$ is thus given by

$$
p_{\alpha}= \begin{cases}\frac{1}{2 \pi}, & \alpha \in[-\pi, \pi), \\ 0, & \text { elsewhere. }\end{cases}
$$

The Doppler frequencies can then be defined by

$$
f=f(\alpha):=f_{m} \cos (\alpha),
$$

where $f_{m}$ is the maximal Doppler frequency. Obviously $f$ is also a random variable. The probability density function of the Doppler frequencies $f$, denoted by $p_{f}(f)$, can be given by [5]

$$
p_{f}(f)= \begin{cases}\frac{1}{\pi f_{m} \sqrt{1-\left(f / f_{m}\right)^{2}}}, & |f| \leq f_{m}, \\ 0, & |f|>f_{m} .\end{cases}
$$

This function of random variables is simulated and shown in Fig. 1 with $f_{m}=20 \mathrm{~Hz}$.

The power spectral density $S_{\mu \mu}(f)$ of the scattered components $\mu(t)=\mu_{1}(t)+j \mu_{2}(t)$, received at the receiving antenna, is obviously proportional to $p_{f}(f)$ of the Doppler frequencies. The following relation holds:

$$
S_{\mu \mu}(f) d f \sim p_{f}(f) d f,
$$

The relation between the probability density function and the power spectrum density is thus established. Further investigation which takes Eq.(3) into account will lead into the Jakes Power spectral density or Clarke power spectral density:

$$
S_{\mu \mu}(f)= \begin{cases}\frac{2 \sigma_{0}^{2}}{\pi f_{m} \sqrt{1-\left(f / f_{m}\right)^{2}}}, & |f| \leq f_{m} \\ 0, & |f|>f_{m} .\end{cases}
$$

where $\int_{-\infty}^{\infty} S_{u u}(f) d f=2 \sigma_{0}^{2}$, and $2 \sigma_{0}^{2}$ is the power of the scattered components $\mu(t)=\mu_{1}(t)+j \mu_{2}(t)$.

The autocorrelation function (ACF) $r_{\mu \mu}(\tau)$ of the scattered component $\mu(t)=\mu_{1}(t)+j \mu_{2}(t)$ can be obtained by taking the inverse Fourier transform of the Jakes power spectral density of Eq.(5):

$$
r_{\mu \mu}(\tau)=2 \sigma_{0}^{2} J_{0}\left(2 \pi f_{m} \tau\right)
$$

where $J_{0}(\cdot)$ is the zeroth-order Bessel function of the first kind.

\section{THE GBHDS CHANNEL MODEL}

In this section we provide a general description for the space-time geometrical-based hyperbolically distributed scatterers (GBHDS) model [6]. This model combined a scalar stochastic fading model for the local scatterers with the geometrical hyperbolic model proposed in [7], [8] for the distribution of the dominant scatterers. The model in [6] assumes that the scatterers are arranged circularly around the mobile, with the distances between 1) the mobile and the local scatterers and 2) the local and dominant scatterers, both being distributed hyperbolically according to an inversecosh-squared distribution. This model provides directional information as well as concerning with mobility issue. Fig.
2 shows the geometry for the GBHDS model. The angle of departure $\psi_{l_{k}}$ is uniformly distributed in the interval $[0,2 \pi]$. The angle $\theta_{l_{k}}$ is the direction of arrival at the base station, while $D$ denotes the distance between the base station and the mobile station. The mobile is located at the origin. This model has the following assumptions [6]

- The scatterers were arranged circularly around the mobile, with the distance between the mobile and the local scatterers $r_{l_{k}}$ and the distance between the local and dominant scatterers $R_{l_{k}}$ are both distributed hyperbolically.

- Signals received at the base station are plane waves propagating along the horizon ( there is no vertical component to the signal propagation).

- Scatterers are omnidirectional re-radiating elements.

- The scatterers have identical scattering coefficients.

- The macrocell antenna heights are relatively high and there is no signal scattering from locations near the base station.

The probability density functions (pdf) of the distances $r_{l_{k}}$ and $R_{l_{k}}$ for the $\mathrm{k}^{\text {th }}$ user in multiuser environment are given by [6]

$$
\begin{array}{r}
f_{r_{l_{k}}}\left(r_{l_{k}}\right)=\frac{a_{1}}{\tanh \left(a_{1} R_{l s}\right) \cosh ^{2}\left(a_{1} r_{l_{k}}\right)} \\
0 \leq r_{l_{k}} \leq R_{l s}
\end{array}
$$

and

$$
\begin{gathered}
f_{R_{l_{k}}}\left(R_{l_{k}}\right)=\frac{a_{2}}{\tanh \left(a_{2} R_{d s}\right) \cosh ^{2}\left(a_{2} R_{l_{k}}\right)} \\
0 \leq R_{l_{k}} \leq R_{d s}
\end{gathered}
$$

and

where $R_{l s}$ is the radius of the circle enclosing the local scatterers, and $R_{d s}$ is the radius of the dominant scatterers circle. The applicable values of $a_{1}$ and $a_{2}$ lie in the interval $(0,1)$. From the spatial probability density functions of the scatterers in (7) and (8) we can determine the the DOA, TOA, and the signal amplitude. A comprehensive study of these models (at theoretical and simulation levels) as well as their validation with practical data have been considered. They proved to be more realistic than other models in the literature when tested against practical data [8], [9].

\section{Statistical Properties of the GBHDS CHANNEL MODEL}

To verify the statistical properties of the GBHDS channel, we first plot the envelope of the Rayleigh process $\mu(t)$, shown in Fig. 3. The elementary properties of $\mu(t)$ such as the the PDF, CDF and the phase of the Rayleigh process $\mu(t)$ are plotted in Fig.4. We simulated $\mu(k T)$ with a sampling interval of $T=0.5 \times 10^{-4} \mathrm{~s}$ and sample number of 8000 . The remaining model parameters were maximum doppler frequency $f_{m}=80 \mathrm{~Hz}$, the carrier frequency $f_{c}=900 \mathrm{MHz}$. 

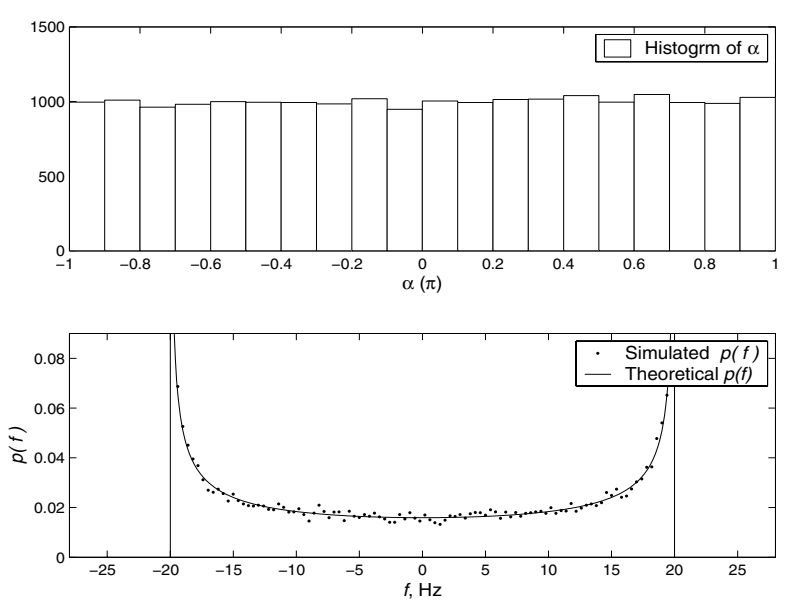

Fig. 1. Histogram of the angles of arrival $\alpha$ and the pdf of the Doppler frequencies $f$.

The autocorrelation (ACF) is also plotted in Fig.5 for the purpose of verification.

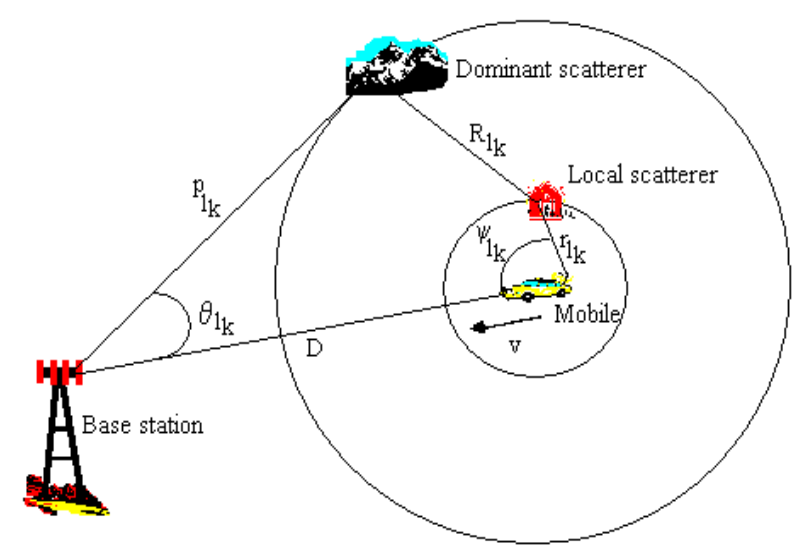

Fig. 2. Geometry of the space-time hyperbolic model.

Apart from the probability density function, the cumulative distribution function, the autocorrelation function, and the knowledge of other statistical quantities that characterize the mobile fading channels like level crossing rate (LCR) and average duration of fades (ADF), is of special interest as it relates the time rate of change of the received signal to the signal level and velocity of the mobile

The level crossing rate, $N_{R}$, is defined as the expected rate at which the envelope crosses a specified signal level, $R$, in the positive direction. In general, it is given by [10]

$$
N_{R}=\int_{0}^{\infty} \dot{r} p(R, \dot{r}) d \dot{r}=\sqrt{2 \pi} f_{m} \rho e^{-\rho^{2}},
$$

where the dot indicates the time derivative and $p(R, \dot{r})$ is the joint density function of $r$ and $\dot{r}$ at $r=R$. And $\rho=R / R_{r m s}$

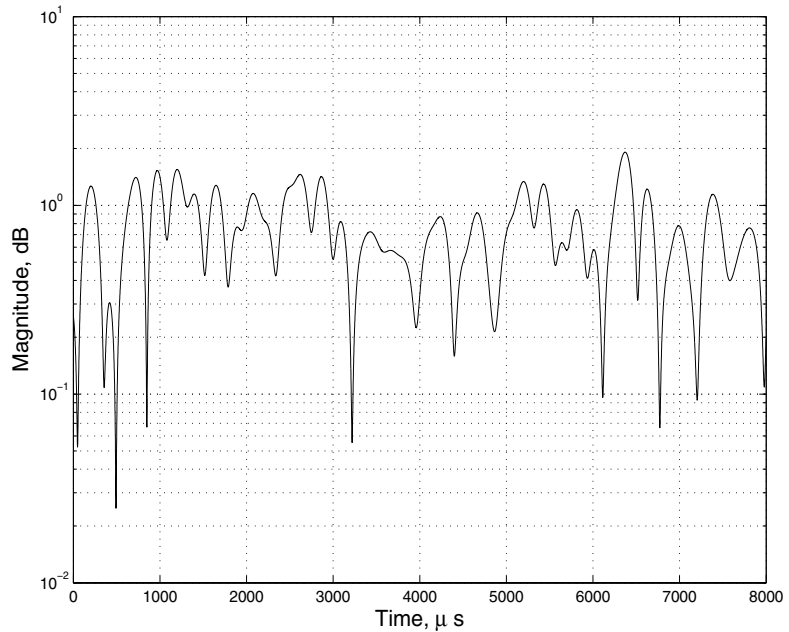

Fig. 3. The Rayleigh envelope of the simulated GBHDS channel.
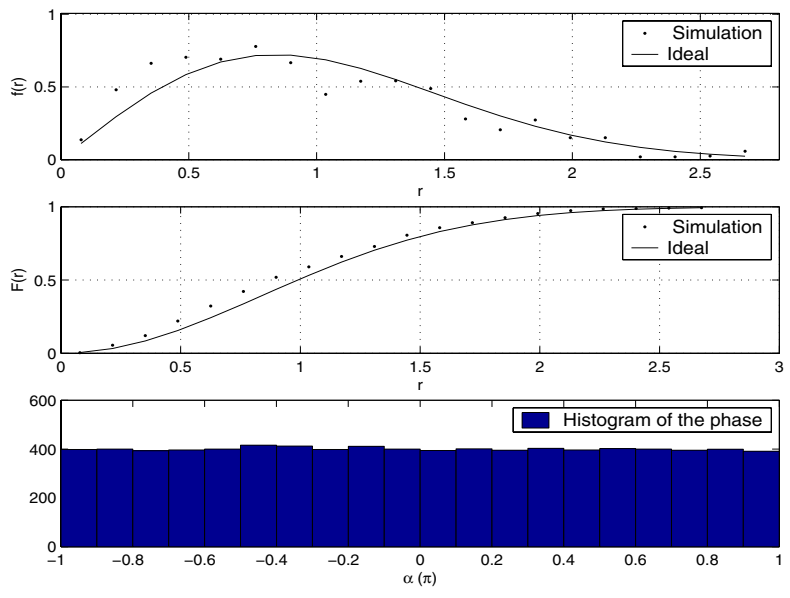

Fig. 4. The PDF and CDF of $\mu(t)$, and the histogram of the fading phase.

is the value of the specified level $R$, normalized to the local rms amplitude of the fading envelope. Fig. 6 shows that the GBHDS model give satisfactory results which are close enough to the ideal curve.

The average duration of fades, $\bar{\tau}$, is defined as the average period of time for which the received signal is below a specified level $R$. Let $\tau_{i}$ be the duration of the $i^{t h}$ fade, then the average duration of fade for a total time interval of length $T$ is $\bar{\tau}=\sum \tau_{i} /\left(N_{R} T\right)$. Like level crossing rate $N_{R}$, the average duration of fade can be also expressed as a function of $\rho$ and $f_{m}$ as [10]

$$
\bar{\tau}=\frac{e^{\rho^{2}-1}}{\rho f_{m} \sqrt{2 \pi}}
$$

Fig. 7 shows the simulated average duration of fade for the above mentioned parameters with the theoretical curve. Again, the simulated curve gives satisfactory results which are close enough to the ideal curve. 


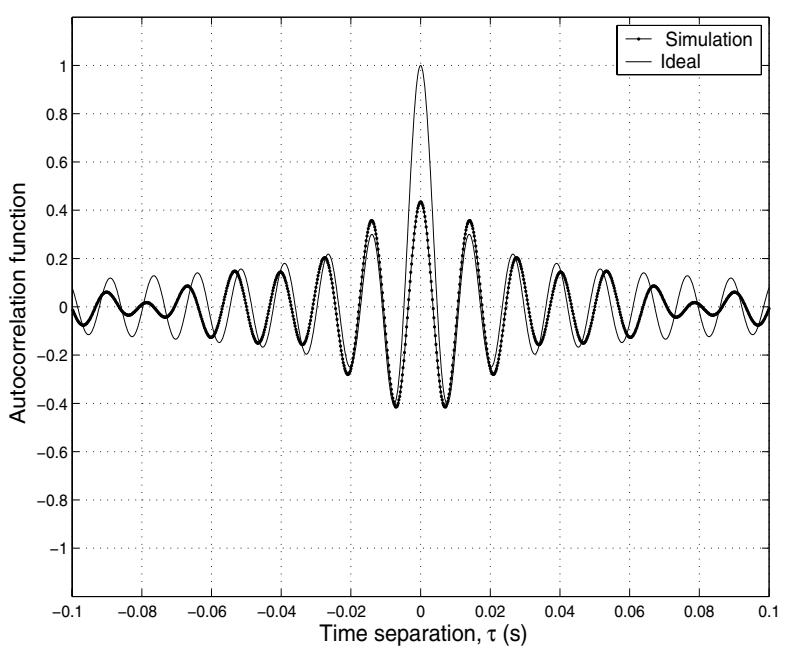

Fig. 5. The ACF of the reference model and the simulation model.

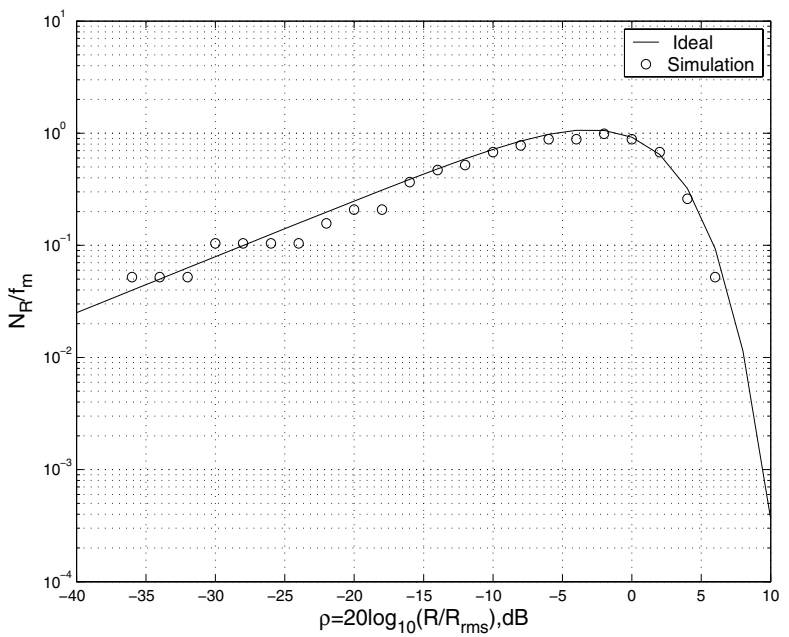

Fig. 6. Normalized level crossing rates of the envelopes.

\section{CONCLUSions}

In this paper, we investigate the GBHDS model's amplitude and phase probability density function (pdf), also the higherorder statistics such as the level crossing rate (LCR) and the average duration of fades (ADF) for the verification against the analytical model. Simulation results show that the simulator of the GBHDS model accurately reproduces all of the important statistical properties. This provides more supports to the GBHDS model and will be useful for simulating practical channels, such as MIMO channels, and space-time-selective mobile fading channels due to the structure of the GBHDS model.

\section{REFERENCES}

[1] M. Patzold, Mobile Fading Channels, John Wiley and Sons, 2002.

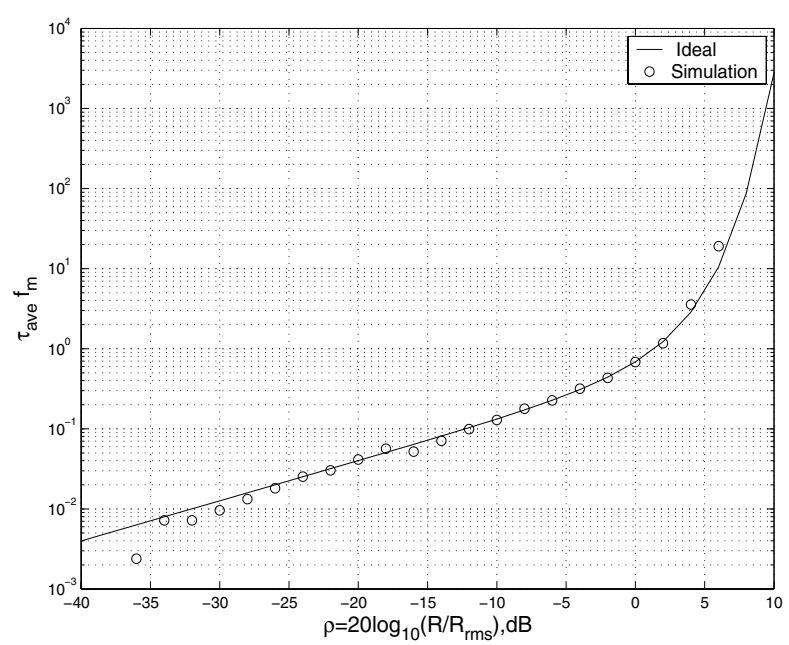

Fig. 7. Normalized durations of fade of the envelopes.

[2] M. Patzold and F. Laue, "Level-Crossing Rate and Average Duration of Fades of Deterministic Simulation Models for Rice Fading Channels," IEEE Trans. Vehicular Technology, vol. 48, no. 4, pp. 1121-1129, July. 1999.

[3] T. S. Rappaport, Wireless Communications, Principles and Practice, Prentice Hall, 2002, 2nd ed.

[4] S. S. Mahmoud, Z. M. Hussain, and P. O'Shea, "A SpaceTime Model for Mobile Radio Channel With Hyperbolically Distributed Scatters," IEEE Antennas and Wireless Propagation Letters, vol. 1, pp. 211-214, 2002.

[5] R. H. Clarke, "A Statistical Theory of Mobile-Radio Reception," Bell Systems Technical Journal, vol. 47, pp. 957-1000, 1968.

[6] Seedahmed S. Mahmoud, Zahir M. Hussain, and Peter O'Shea, "Space-time model for mobile radio channel with hyperbolically distributed scatterers," IEEE Antennas and Wireless Propagation Letters, vol. 1, no. 12, pp. 211-214, 2002.

[7] — " "Geometrical model for mobile radio channel with hyperbolically distributed scatterers," The 8th IEEE International Conference on Communications Systems, vol. 1, pp. 1720, Singapore, Nov. 2002.

[8] _ - "A Geometrical-Based Channel Model with Hyperbolically Distributed Scatterers for a Macrocell Mobile Environment with Antenna Array," Multimedia Cyberscape Journal, vol. 2, pp.1-10, 2004.

[9] _ , "Spatial and Temporal Statistics for the GeometricalBased Hyperbolic Macrocell Channel Model," Submitted to IEEE Transactions on Vehicular Technology.

[10] W. C. Jakes, Microwave Mobile Communications, Wiley, New York, 1974. 